\title{
Establishment of New Fundamental Theory in Diffusion Phenomena
}

\author{
Takahisa Okino $^{1}$ \\ ${ }^{1}$ Applied Mathematics Department, Faculty of Science \& Engineering, Oita University, Oita, Japan \\ Correspondence: Takahisa Okino, Applied Mathematics Department, Faculty of Science \& Engineering, Oita \\ University, Oita, Japan. E-mail: okino@oita-u.ac.jp
}

Received: January 4, 2019

Accepted: January 21, 2019

Online Published: January 29, 2019

doi:10.5539/apr.v11n1p82

URL: http://dx.doi.org/10.5539/apr.v11n1p82

\begin{abstract}
Investigating the elementary process of diffusion revealed that the De Broglie hypothesis is really valid in a material and further that the Schrödinger equation is reasonably derived from the diffusion equation. The diffusion equation is thus one of the fundamental equations in physics. The problem between a moving coordinate system and a fixed coordinate system for the diffusion equation had never been discussed until recently in the history of diffusion theory. In that situation, it is revealed that investigating the problem between those coordinate systems is indispensable for understanding the diffusion phenomena. The new findings obtained here, which are revolutionary in the existing diffusion theory, will be not only dominant but also indispensable for further advance in the diffusion study.
\end{abstract}

Keywords: Diffusion Equation, Diffusivity, Fick's Laws, Markov Process

\section{Introduction}

The solutions of thermal conduction equation proposed by Fourier show that the temperature distribution in a material satisfies the parabolic law (Fourier, 1822). Based on the fact that the concentration distribution of micro particles resulting from the diffusion experiments also satisfies the parabolic law, Fick proposed that the Fourier's thermal conduction equation is applicable to diffusion phenomena as it is (Fick, 1855). In that case, the temperature and the coefficient of thermal conductivity in a material were rewritten into the concentration $C(t,|r\rangle)$ of micro particles and the diffusivity $D$ in the time $t$ and space $\langle r|=(x, y, z)$. Under the condition of nonexistence of the sink and source relevant to micro particles concerned, the diffusion flux $\left|J_{\mathrm{F}}\right\rangle$ and diffusion equation given by

$$
\begin{gathered}
\left|J_{\mathrm{F}}\right\rangle=-D|\nabla\rangle C(t,|r\rangle) \\
\partial_{t} C(t,|r\rangle)=D\langle\tilde{\nabla} \mid \nabla\rangle C(t,|r\rangle)
\end{gathered}
$$

have been thus accepted as the Fick's first law and the Fick's second law, corresponding to the thermal flux and thermal conduction equation. In those days, the diffusivity $D$ was conceived as a physical constant corresponding to the coefficient of thermal conductivity. In Equations (1) and (2), the Hermite conjugate $\langle\tilde{\nabla}|=-| \nabla\rangle^{\dagger}=\left(\partial_{x}, \partial_{y}, \partial_{z}\right)$ relevant to the Dirac bracket and the abbreviate notation $\partial_{\xi}=\partial / \partial \xi$ are used.

After the Fick's proposition, the extended Fick's second law given by

$$
\partial_{t} C(t,|r\rangle)=\langle\tilde{\nabla} \mid D \nabla\rangle C(t,|r\rangle)
$$

has been also accepted as a law applicable to such a case that $D$ depends on $C(t,|r\rangle)$. When $D$ is acceptable as a physical constant, Equation (3) is naturally consistent with Equation (2).

The temperature distribution is a physical quantity relevant to the thermal state quantity. On the other hand, the concentration distribution is a real quantity of micro particles in a material. The diffusion is caused by the exchange of position between a micro particle and a micro hole, which is composed of a vacancy and/or an interstice among micro particles, for each other. It is thus considered that the space of diffusion region moves relatively against the diffusion region outside. In fact, the diffusion flux of vacancies is conceived in the existing 
diffusion theory and also the experimental result of Kirkendall effect shows that the diffusion region space moves against the diffusion region outside (Kirkendall, 1947).

The problem between a moving coordinate system and a fixed coordinate system for the diffusion equation had never been discussed until recently in the history of diffusion theory. However, it is obvious that the relative motion of micro particles and micro holes in the diffusion region inside is expressed by such a moving coordinate system that the coordinate origin is set at a point in the space of diffusion region (Okino, 2014; Okino, Cho, \& Yamada, 2017; Cho, Yamada, \& Okino, 2018). On the other hand, the experimental data correspond to the solutions of diffusion equation expressed by a fixed coordinate system in the diffusion region outside because of the experimental observation outside the diffusion region. Therefore, the discussion about the coordinate transformation is indispensable for investigating the analytical solutions and the experimental results.

Recently, the elementary process of diffusion and the coordinate transformation of diffusion equation were investigated and some dominant findings were obtained then (Okino, 2011, 2013, 2015, 2018). In those findings, it is a revolutionary finding that the diffusion Equation (2) is valid in a moving coordinate system, even if $D$ depends on $C(t,|r\rangle)$. On the other hand, Equation (3) is expressed as a fixed coordinate system in relation to the continuous equation. This means that the difference between solutions of Equations (2) and (3) is only a phase shift between their solutions resulting from the relative velocity between the coordinate origins of the moving and fixed coordinate systems used for Equations (2) and (3). Equation (2) is thus mathematically equivalent to Equation (3). It is obvious that analyzing Equation (2) is superior in calculation to Equation (3) (Okino, 2011, 2018).

In history, the diffusion flux had been accepted as a law proposed by Fick for a long time. In theory, however, the diffusion flux should be determined by integrating the continuous equation with respect to the space. The Fick's first law is thus incomplete without an integral constant relevant to the integral calculation. Einstein used the Fick's first law in the theory of Brown motion (Einstein, 1905). The paradox caused by using the Fick's first law in the Einstein's Brown theory will be clarified using the new diffusion theory in the text.

Investigating the Markov process (Markov, 1960) reveals that Equation (2) is valid in a moving coordinate system, even if $D$ depends on $C(t,|r\rangle)$ (Okino, 2018). Subsequently, Equation (3) is reasonably obtained by the coordinate transformation of Equation (2). Further, the continuous equation shows that the diffusion flux is reasonably determined from Equation (3) obtained here.

There is often such a case that a relation is universally valid under a given condition in accordance with an empirical rule in physics. If we cannot theoretically demonstrate its validity then, it has been accepted as a law in physics. We can hardly encounter such a matter that a physical law having been accepted as a reasonable one for a long time is actually not in conformity with the condition of a law in physics. As mentioned above, it will be concretely revealed in the following that the Fick's second law as well as the Fick's first law does not satisfy the condition of a law in physics. At the same time, some new findings resulting from the coordinate transformation theory of the diffusion equation, which are not only dominant but also indispensable for further advance of the diffusion study, are discussed in accordance with the fundamental theory in physics.

\section{Diffusion Equation of a Moving Coordinate System}

The coordinate origin of $(t,|r\rangle)$ is set at an arbitrary point of space in a material. Using the normalized concentration $C(t,|r\rangle)$ of an element in a material, the relation of

$$
C(t+\Delta t,|r\rangle)=\{C(t,|r\rangle+|\Delta r\rangle)+C(t,|r\rangle-|\Delta r\rangle)\} / 2
$$

is valid in accordance with the Markov process (Markov, 1960). The diffusion equation of

$$
\partial_{t} C=D\langle\tilde{\nabla} \mid \nabla\rangle C \text { for } D=(\Delta r)^{2} / 2 \Delta t
$$

is obtained by the Taylor expansion of the above relation (Okino, 2013). In general, the diffusivity $D$ of Equation (2') depends on $C(t,|r\rangle)$, because of the arbitrariness of a coordinate origin set in the diffusion region space. The concept of vacancy flux in theory and the Kirkendall effect in experimentation show that the diffusion region space moves (Kirkendall, 1947). It is, therefore, obvious that Equation (2') is valid in a moving coordinate system regardless of whether $D$ depends on $C(t,|r\rangle)$ or not (Okino, 2018).

The Schrödinger equation (Schrödinger, 1026), which is the most important one in the quantum mechanics, was derived from using the De Broglie hypothesis of the matter wave (De Broglie, 1923). The Schrödinger equation 
was also derived from investigating the elementary process of diffusion, regardless of the De Broglie hypothesis (Okino, 2013). The universal expression of diffusivity, which is applicable to a material in the arbitrary thermal state, given by

$$
D=5 \times 10^{-8} \frac{N_{\mathrm{A}} \hbar}{n} \exp \left[\frac{U-Q}{k_{\mathrm{B}} T}\right] \quad\left[m^{2} s^{-1}\right]
$$

was obtained by using $D$ of Equation (2') then (Okino, 2018). In Equation (4), $\exp \left[-Q / k_{\mathrm{B}} T\right]$ is the well-known Boltzmann factor in a material with an activation energy $Q$ at an absolute temperature $T$ (Boltzmann, 1872), and $U$ is a potential energy relevant to such a force $F(=-\nabla U)$ as a driving force and/or an external force acting on micro particles in the diffusion field concerned, including an entropy effect $U_{0}=S T$. Further, $\hbar$, $N_{A}$ and $n$ are the Planck constant, the Avogadro constant and a molecular weight. In the actual case, $D$ of Equation (2') should be thus replaced by that of Equation (4).

\section{Diffusion Equation of a Fixed Coordinate System}

Applying a differentiable vector $\mid J(t,|r\rangle)\rangle$, which is a flux of physical quantity $C(t,|r\rangle)$ per unit time and unit square, to the Gauss theorem yields the continuous equation of

$$
\partial_{t} C+\langle\tilde{\nabla} \mid J\rangle=0
$$

in time $t$ and space $\langle r|=(x, y, z)$ under the condition of nonexistence of sink and source relevant to the physical quantity.

The extended Fick's second law has been accepted as

$$
\partial_{t} C=\langle\tilde{\nabla} \mid D \nabla\rangle C
$$

in the diffusion theory. Taking account of the Fick's first law of Equation (1), we define the new diffusion flux given by

$$
|J\rangle=\left|J_{\mathrm{F}}\right\rangle+|J(t)\rangle+\left|J_{\text {eq }}\right\rangle
$$

where the diffusion flux $|J(t)\rangle$ depends only on $t$ and the diffusion flux $\left|J_{\text {eq }}\right\rangle$ does not depend on $(t, x, y, z)$. In other words, $|J(t)\rangle+\left|J_{\text {eq }}\right\rangle$ of Equation (6) corresponds to a mathematical integral constant of the differential Equation (5) (Okino, 2015). Equation (3) is then reasonably obtained by substituting Equation (6) into Equation (5) because of $\langle\tilde{\nabla} \mid J(t)\rangle=\left\langle\tilde{\nabla} \mid J_{\text {eq }}\right\rangle=0$. Here, note that there is no such concept as a moving coordinate system in the Gauss theorem. This means that Equation (5) is expressed as a fixed coordinate system. Therefore, Equation (3) obtained here is also expressed as a fixed coordinate system.

\section{Coordinate Transformation Theory of Diffusion Equation}

Here, we define a moving coordinate system $\left(t^{\prime},\left|r^{\prime}\right\rangle\right)$ and a fixed coordinate one $(t,|r\rangle)$ under the initial condition of $\left|r^{\prime}\right\rangle=|r\rangle=0$ at $t^{\prime}=t=0$. Substituting the relations of differential operators $\partial_{t^{\prime}}=\partial_{t}+\langle v \mid \nabla\rangle$ and $\nabla^{\prime}=\nabla$, which are obtained by using a relative velocity $|v\rangle$ between the coordinate origins of $\left(t^{\prime},\left|r^{\prime}\right\rangle\right)$ and $(t,|r\rangle)$, into Equation (2') of the moving coordinate system $\left(t^{\prime},\left|r^{\prime}\right\rangle\right)$ yields the diffusion equation of the fixed coordinate system given by

$$
\partial_{t} C(t,|r\rangle)=D\langle\tilde{\nabla} \mid \nabla\rangle C(t,|r\rangle)+\langle v \mid \nabla\rangle C(t,|r\rangle)
$$

The relation $\partial_{\Delta r} D=v_{\mathrm{r}}(=\Delta r / \Delta t)$ obtained by differentiating $D$ in Equation (2') indicates that the gradient of diffusivity corresponds to a velocity of diffusion particle in the local space $d V=d x d y d z$, i.e., $\langle\tilde{\nabla}| D=-\langle F| D / k_{\mathrm{B}} T=-\left\langle v_{\mathrm{F}}\right|$ resulting from the derivative of Equation (4), using the Hermite conjugate 
$\left\langle v_{\mathrm{F}}|=-| v_{\mathrm{F}}\right\rangle^{\dagger}$. In a given local space, the jumping frequency of a diffusion particle is statistical. In the diffusion region at a given time, the diffusion space moves with a velocity $|v\rangle$ as a whole, corresponding to jumping of diffusion particles in the diffusion region, because of the continuousness and no mass of diffusion region space composed of micro holes.

In the experimentation of diffusion, under the condition of being able to neglect the shape variation of the diffusion specimen, the total number of micro particles on a cross section perpendicular to a proceeding direction of diffusion is conserved with good approximation. In that case, using the normalized concentration $C^{j}$ $(j=1,2, \cdots, M)$ for $M$ elements on the cross section, the relation of

$$
\sum_{j=1}^{M} C^{j}=1
$$

is usually accepted. Under the condition of Equation (8), it was revealed that the relation between the diffusivities relevant to the $M$ elements given by

$$
\tilde{D}=D^{1}=D^{2}=\cdots=D^{M}
$$

is valid as a mathematical operator in the differential equation (Okino, 2014, 2015). This means that the above differential equations without the suffix $j$ hold for each of $M$ elements in the diffusion region by replacing $D$ with $\tilde{D}$.

The one-to-one correspondence of the positional exchange between a diffusion particle and a micro hole results in the relation of

$$
\langle v|=-\left\langle v_{\mathrm{F}}\right|=\langle\tilde{\nabla}| D=-\langle F| D / k_{\mathrm{B}} T
$$

in the differential equation. Substituting Equation (10) into Equation (7) yields

$$
\partial_{t} C(t,|r\rangle)=D\langle\tilde{\nabla} \mid \nabla\rangle C(t,|r\rangle)+\langle\tilde{\nabla} D \mid \nabla\rangle C(t,|r\rangle)=\langle\tilde{\nabla} \mid D \nabla\rangle C(t,|r\rangle)
$$

By using the diffusion Equation (2') derived from the Markov process, the extended Fick's second law expressed by Equation (3) is reasonably obtained here. At this point, the Fick's second law becomes baseless as a law in physics.

Equation (10) gives the evidence that micro particles randomly move in a material even if such a force $F$ as a driving force and/or an external one acts on them. Therefore, Equation (10) resulting from the coordinate transformation theory has an important concept in the diffusion phenomena. The diffusion flux relevant to Equation (3) expressed by a fixed coordinate system corresponds to Equation (6). The inverse transformation from Equation (3) into Equation (2') is naturally possible (Okino, 2018). The diffusion flux relevant to Equation (2') yielding

$$
|J\rangle=\left|J_{\mathrm{F}}\right\rangle+|v\rangle C(t,|r\rangle)+\left|J_{\text {eq }}\right\rangle
$$

is a moving coordinate system (Okino, 2015).

In the previous work (Okino, 2014; Okino, Cho, \& Yamada, 2017; Cho, Yamada, \& Okino, 2018), the Kirkendall effect was reasonably understood by using the diffusion flux $|J(t)\rangle$ of diffusion region space for interdiffusion problems. Further, it was also revealed that the existence of $|J(t)\rangle$ causes the random movement of micro particles in the diffusion field, even if an external force as well as a driving force acts on micro particles, regardless of the force orientation. The intrinsic diffusion flux $\left|J_{\text {eq }}\right\rangle$ is an indispensable concept for understanding a self-diffusion problem satisfying $|\nabla\rangle C=0$ and $|J(t)\rangle=0$ or $|v\rangle=0$ in Equation (6) or (11) (Okino, 2015). The Fick's first law is thus physically incomplete without an initial and/or boundary value $|J(t)\rangle+\left|J_{\text {eq }}\right\rangle$ for the differential Equation (5). In other words, it is obvious that the Fick's first law does not have the universality as a law in physics.

Taking no account of the problem of coordinate system for the diffusion flux, Einstein derived the diffusivity of

$$
D=k_{\mathrm{B}} T / k
$$


from using the relations of diffusion flux $|J\rangle=|v\rangle C$ and force $|F\rangle=k|v\rangle$ relevant to the velocity $|v\rangle$ in analyzing the Brown motion (Einstein, 1905). Here, substituting $|J\rangle=|v\rangle C$ into Equation (5) yields not the diffusion equation but the Euler equation in the fluid mechanics. That is inconsistent with the theory itself. For the paradox, if $J=|| J\rangle \mid$ and $v=\| v\rangle \mid$ is assumed to be $\lim _{\nu \rightarrow 0}\{J / v C\}=1$ in the Einstein theory, Equation (12) is valid in the thermal equilibrium state then. However, it is obvious in the present theory that Equation (12) is reasonably obtained by substituting $|F\rangle=k|v\rangle$ into Equation (10) and is valid in the arbitrary state during diffusion then.

\section{Conclusions and Discussion}

Investigating the elementary process of diffusion revealed that the diffusion equation is an essential equation not only in the diffusion phenomena but also in the fundamental physics in relation to the matter wave and the Schrödinger equation (Okino, 2013, 2018). The diffusion equation as well as the diffusion flux has been accepted as a law proposed by Fick in 1855 until now. In the present work, however, it was revealed that the Fick's first law does not have the universality as a law in physics and that the extended Fick's second law is reasonably derived from the Markov process. It is thus obvious that the Fick's laws are not in conformity with the condition of a law in physics. Hereafter, it will be thus unsuitable that the phrases of the Fick' first law and Fick's second law are used for the diffusion flux and diffusion equation.

The descriptions relevant to the Fick's laws are widely found in a lot of textbooks, technical journals and books, encyclopedias, electric media, and so on. The matter itself causes such a serious and social problem that a lot of people will be continuously influenced by the wrong descriptions in the published matters, if we leave it unsolved. Thus, we have a responsibility to solve the problem for a lot of people. First of all, making the matter universally known for specialists in physics as soon as possible should be indispensable for solving the problem.

Hereinbefore, the new fundamental theory in diffusion phenomena was established in accordance with the coordinate transformation theory for the diffusion equation. At the same time, it was revealed that Equation (3) is mathematically equivalent to Equation (2'). Hereafter, Equation ( 2 '), which is superior in calculation to analyzing Equation (3), will be thus widely used as a fundamental equation for analyzing various diffusion problems.

\section{Conflict of interests}

The authors declare that there is no conflict of interests regarding the publication of this paper.

\section{References}

Fourier, J. B. J. (1822). Theorie analytique de la chaleur. Didot Paris, 499-508.

Fick, A. (1855). On liquid diffusion. Philosophical Magazine and Journal of Science, 10, 31-39.

Smigelskas, A. D., \& Kirkendall, E. O. (1947). Zinc diffusion in alpha brass. Transactions of AIME, 171, 130-142.

Okino, T. (2014). Diffusion Behavior and Brown Motion in Many Elements System. Applied Physics Research, 6, $1-7$.

Okino, T., Cho, H., \& Yamada, M. (2017). Fundamental Concept of Interdiffusion Problems. Journal of Modern Physics, 8, 903-918.

Cho, H., Yamada, M., \& Okino, T. (2018). New Analytical Method of Interdiffusion Problems. Journal of Modern Physics, 9, 130-144.

Okino, T. (2011). New Mathematical Solution for Analyzing Interdiffusion Problems. Materials Transactions, 52, 2220-2227.

Okino, T. (2013). Correlation between Diffusion Equation and Schrödinger Equation. Journal of Modern Physics, $4,612-615$.

Okino, T. (2015). Mathematical Physics in Diffusion Problems. Journal of Modern Physics, 6, 2109-2144.

Okino, T. (2018). Quantum Effect on Elementary Process of Diffusion and Collective Motion of Brown Particles. Journal of Modern Physics, 9, 1007-1028.

Einstein, A. (1905). Die von der molekularkinetishen theorie der warme geforderte bewegung von in ruhenden flussiigkeiten sspendierten teilchen. Annalen der Physik, 17, 549-560.

Markov, A. A. (1960). The theory of algorithms. American Mathematical Society Translations Series, 15(2), 1-14.

Schrödinger, E. (1926). Quantisierung als eigenvertproblem. Annalen der Physik, 79, 361-376.

De Broglie, L. (1923). Wave and quanta. Nature, 112, 540. 
Boltzmann, L. (1872). Weitere studien uber das warmegleichwicht unter gasmolekuen. Wiener Berichte, 66, 275-370.

\section{Appendix}

The errata of papers written before 2017 are reported at the Appendix in Ref. [Cho, H., Yamada, M., \& Okino, T. (2018)]. The erratum of Ref. [Cho, H., Yamada, M., \& Okino, T. (2018)] is as follows.

$$
\text { P. 139, Equation (27) } \rightarrow m=\frac{2}{\mu}\left|\left(\sqrt{D_{\mathrm{A}}^{\mathrm{Cu}}}-\sqrt{D_{\mathrm{B}}^{\mathrm{Zn}}}\right)\left(C_{\mathrm{A}}^{\mathrm{Zn}}-C_{\mathrm{B}}^{\mathrm{Zn}}\right)\right| .
$$

The errata at the pages 1019 and 1020 in Ref. [Okino, T. (2018)] are as follows.

$$
F_{x} \ll(\text { or }>>) k_{\mathrm{B}} T\left|\partial_{x}{ }^{2}(t, x) / \partial_{x} C(t, x)\right| / D \rightarrow F_{x} \ll(\text { or }>>) k_{\mathrm{B}} T\left|\partial_{x}{ }^{2} C(t, x) / \partial_{x} C(t, x)\right| .
$$

\section{Copyrights}

Copyright for this article is retained by the author(s), with first publication rights granted to the journal.

This is an open-access article distributed under the terms and conditions of the Creative Commons Attribution license (http://creativecommons.org/licenses/by/4.0/). 